\title{
Phase-field modeling of the beta to omega phase transformation in $\mathrm{Zr}-\mathrm{Nb}$ alloys
}

\author{
Hemantha Kumar Yeddu ${ }^{\mathrm{a}, *}$, Turab Lookman ${ }^{\mathrm{a}}$ \\ ${ }^{a}$ Theoretical Division, Los Alamos National Laboratory, Los Alamos, NM 87545, USA.
}

\begin{abstract}
A three dimensional elastoplastic phase-field model is developed, using the Finite Element Method (FEM), for modeling the athermal beta to omega phase transformation in $\mathrm{Zr-Nb}$ alloys by including plastic deformation and strain hardening of the material. The microstructure evolution during athermal transformation as well as under different stress states, e.g. uni-axial tensile and compressive, bi-axial tensile and compressive, shear and tri-axial loadings, is studied. The effects of plasticity, stress states and the stress loading direction on the microstructure evolution as well as on the mechanical properties are studied. The input data corresponding to a $\mathrm{Zr}-8$ at.\% $\mathrm{Nb}$ alloy are acquired from experimental studies as well as by using the CALPHAD method. Our simulations show that the four different omega variants grow as ellipsoidal shaped particles. Our results show that due to stress relaxation, the athermal phase transformation occurs slightly more readily in the presence of plasticity compared to that in its absence. The evolution of omega phase is different under different stress states, which leads to the differences in the mechanical properties of the material. The variant selection mechanism, i.e. formation of different variants under different stress loading directions, is also nicely captured by our model.
\end{abstract}

Keywords: Phase-field method, diffusionless phase transformation, omega phase, microstructure, zirconium-niobium alloys

\section{Introduction}

Zirconium (Zr)-based alloys have been widely used in the nuclear and chemical industries due to their unique properties. The recent advances show that Zr-based alloys can also be used in medical implants [1] and as a crucial component in the preparation of bulk metallic glasses [2]. The occurrence of a phase transformation in a Zr-based product in service will significantly influence the mechanical properties of the product. More importantly, the metastable omega phase, a highly brittle phase, can form in $\mathrm{Zr}$-alloys under various thermomechanical conditions and could even lead to failure of the product.

*Corresponding Author. E-mail: hemy@lanl.gov, hemu23@gmail.com

Preprint submitted to Materials Science and Engineering A

December 22, 2014 
The three phases that mainly form in Zr-alloys are $\alpha, \beta$ and $\omega$ with crystal structures hexagonal close packed (hcp), body centered cubic (bcc) and hexagonal, respectively. The omega $(\omega)$ phase can form from alpha phase $(\alpha)$ under high static pressure or during shock loading in pure Zr $[3,4]$. The $\omega$ phase can also form from beta $(\beta)$ phase either athermally (by rapid quenching or stressinduced) or isothermally (by holding the alloy at a constant temperature below $773 \mathrm{~K})[4,5]$. The athermal phase transformation is a diffusionless transformation, whereas the isothermal transformation is diffusion-controlled [4]. However, both athermal and isothermal transformations are first order solid state phase transformations.

The athermal $\beta \rightarrow \omega$ phase transformation is a shuffle driven transformation that takes place by means of a lattice collapse mechanism. The ideal $\omega$ lattice can be obtained from a bcc lattice by collapsing one pair of $(111)^{b c c}$ planes and by keeping the neighboring $(111)^{b c c}$ plane and its periodic replica unaltered [5]. The athermal $\beta \rightarrow \omega$ phase transformation gives rise to a microstructure consisting of fine omega particles, with a typical size range of $2-10 \mathrm{~nm}$. The omega particles can form in four different crystallographic orientations (variants), homogeneously distributed in the parent $(\beta)$ phase and aligned along one of the $\langle 111\rangle_{\beta}$ directions. This morphology is different from the plate-like morphology of martensite and hence the athermal $\beta \rightarrow \omega$ phase transformation is not classified as a martensitic transformation, despite its athermal and diffusionless nature [3].

As the formation of fine brittle omega particles can vary the mechanical properties of the material, it is essential to study the co-evolution of microstructure and properties during the athermal $\beta \rightarrow \omega$ phase transformation in $\mathrm{Zr}-\mathrm{Nb}$ alloys under different thermo-mechanical conditions using in-situ techniques. However, due to the difficulties associated with experimental set-ups to study the phase transformation under complex stress states, the capabilities of in-situ measurement techniques are limited.

Although constitutive models have been used to study the relation between phase transformation and plasticity $[6,7]$, the recent advances in modeling the microstructure evolution by using the phase-field method $[8,9]$ are promising and can aid in the study of the beta to omega phase transformation. Several studies on solid state phase transformations using the phase-field approach have advanced this research area [10-30]. The development of the Ginzburg-Landau model coupled with Microelasticity theory, i.e. Phase-field microelasticity theory proposed by Khachaturyan [10], is an important advancement in this field. An alternative approach is developed by using the Ginzburg-Landau formulation in terms of strain order parameters and by enforcing the compatibility condition of strains to ensure that the dispalcement field is continuous $[12,18]$. This is in contrast to solving the displacement equations in terms of non-strain order parameters, as proposed by Khachaturyan [10]. Another important advancement is the inclusion of plasticity within the phase-field approach [11, 19, 22, 23, 31], as it is essential to study the interplay between the martensitic transformation, internal stresses, strains and plastic strains in order to understand the relationships between the microstructure evolution and the mechanical properties of the 
material.

In the present work a three dimensional (3D) phase-field model is developed by including plasticity and strain hardening within the Phase-field microelasticity theory $[11,19,23]$. The diffusionless athermal beta (bcc) to omega (hexagonal) phase transformation occurring in a single crystal of a $\mathrm{Zr}-8$ at. $\% \mathrm{Nb}$ alloy is studied. The finite element method, which allows a straight forward and transparent formulation of the equations, is applied to solve the phase-field as well as the continuum mechanics equations. The effects of plasticity, stress states and stress loading directions on the omega phase formation are studied. Different stress states (e.g. uni-axial tensile and compressive, bi-axial tensile and compressive, shear and tri-axial loadings) are considered in order to study their effects on the microstructure evolution as well as on the mechanical properties of the material. Our results indicate that the phase transformation occurs slightly more readily in the presence of plasticity. Different stress states as well as the loading directions play an important role in the microstructure evolution as well as affect the mechanical properties of $\mathrm{Zr}-\mathrm{Nb}$ alloys.

\section{Elastoplastic phase-field model}

A diffusionless phase transformation can be modeled using the Cahn-Allen equation [32], also known as the Time-Dependent Ginzburg-Landau kinetic equation [10]. The microstructure evolution can be simulated by predicting the time-dependent variation of the phase-field variable $\eta_{p}$, which in turn is related to the minimization of the Gibbs energy $G$ of the system with respect to the phase-field variable as:

$$
\frac{\partial \eta_{p}}{\partial t}=-\sum_{q=1}^{q=v} L_{p q} \frac{\delta G}{\delta \eta_{q}}
$$

where $\frac{\delta G}{\delta \eta_{q}}$ is a variational derivative that serves as a driving force for the formation of the product phase denoted by the phase-field variable $\eta_{q}$, which is dependent on the position vector $\mathbf{r}$. $v$ is the total number of crystallographic orientations of the product phase and $L_{p q}$ is a kinetic parameter.

The phase transformation of beta $(\beta)$ phase (body centered cubic structure, bcc) in to omega $(\omega)$ phase (hexagonal structure) causes asymmetry in the crystal structure and thereby gives rise to four possible crystallographic orientations (variants) of $\omega$. Thus, in order to represent the four possible variants, four phase-field variables, i.e. $\eta_{1}, \eta_{2}, \eta_{3}, \eta_{4}$, need to be considered in the model. Hence four phase-field equations, i.e. for $p=1,2,3,4$ and $v=4$ in Eq. (1), need to be solved at each time step. Each of the above four variants is governed by a stress-free transformation strain tensor $\epsilon_{i j}^{0}(p)$.

From a thermodynamic point of view, the Gibbs energy of a system undergoing athermal phase transformation consists of the following parts:

$$
G=\int_{V}\left(G_{v}^{\text {chem }}+G_{v}^{\text {grad }}+G_{v}^{e l}\right) d V
$$


$G_{v}^{\text {chem }}$ corresponds to the chemical part of the Gibbs energy density of an unstressed system at the temperature under consideration. $G_{v}^{\text {grad }}$ is the extra Gibbs energy density caused by the interfaces. $G_{v}^{e l}$ is the elastic strain energy density, generated due to the structural phase transformation of $\beta \rightarrow \omega$.

\subsection{Chemical energy}

The chemical part of the Gibbs energy density $G_{v}^{c h e m}$, expressed as a Landautype polynomial $[11,15]$, is given by:

$$
\begin{aligned}
& G_{v}^{\text {chem }}\left(\eta_{1}, \eta_{2}, \eta_{3}, \eta_{4}\right)=\frac{1}{V_{m}}\left[\frac{1}{2} A\left(\eta_{1}^{2}+\eta_{2}^{2}+\eta_{3}^{2}+\eta_{4}^{2}\right)\right. \\
& \left.-\frac{1}{3} B\left(\eta_{1}^{3}+\eta_{2}^{3}+\eta_{3}^{3}+\eta_{4}^{3}\right)+\frac{1}{4} C\left(\eta_{1}^{2}+\eta_{2}^{2}+\eta_{3}^{2}+\eta_{4}^{2}\right)^{2}\right]
\end{aligned}
$$

By considering the driving force $\Delta G_{m}$, i.e. the difference in the Gibbs energies of beta and omega phases, and the Gibbs energy barrier $\Delta G^{*}$ terms in the above equation, the coefficients are modified [11] as: $A=32 \Delta G^{*}$, $B=3 A-12 \Delta G_{m}$ and $C=2 A-12 \Delta G_{m}$. Here $\Delta G^{*}=\frac{V_{m} \beta^{\prime}}{2 \delta^{2}}$, where $V_{m}$ is molar volume, $\delta$ is thickness of the interface and $\beta^{\prime}$ relates to interfacial energy and is discussed in the following section.

\subsection{Gradient Energy}

The gradient energy density term, $G_{v}^{\text {grad }}$ can be expressed as $[11,15]$ :

$$
G_{v}^{g r a d}=\frac{1}{2} \sum_{p=1}^{p=v} \beta_{i j}(p) \frac{\partial \eta_{p}}{\partial r_{i}} \frac{\partial \eta_{p}}{\partial r_{j}}
$$

where $\mathbf{r}(\mathbf{x}, \mathbf{y}, \mathbf{z})$ is the position vector expressed in cartesian coordinates, $\beta_{i j}$ is the gradient coefficient matrix expressed in terms of the interfacial energy $\gamma$, molar volume $V_{m}$ and Gibbs energy barrier $\Delta G^{*}$. In the present work isotropic interfacial properties are considered, i.e. $\beta_{i j}$ is considered as a diagonal tensor with all the elements equal to $\beta^{\prime}[11]$, given as:

$$
\beta^{\prime}=\frac{9 \gamma^{2} V_{m}}{16 \Delta G^{*}}
$$

\subsection{Elastic energy}

In order to calculate the elastic strain energy density $G_{v}^{e l}$, the elastic stress needs to be calculated by using the Microelasticity theory [10], which is briefly explained below. A diffusionless displacive transformation gives rise to deformation of the crystalline lattice and thereby induces stress-free transformation strains $\epsilon_{i j}^{0}(\mathbf{r})$ into the material. The surrounding parent matrix exerts strain $\epsilon_{i j}(\mathbf{r})$ on the product phase to resist the stress-free transformation strains and thereby induces elastic strain $\epsilon_{i j}^{e l}(\mathbf{r})$, which in turn gives rise to elastic stress $\sigma_{i j}(\mathbf{r})$, in the material. When the elastic stress exceeds the yield limit of the 
material, plastic deformation initiates and gives rise to plastic strain $\epsilon_{i j}^{p l}(\mathbf{r})$. The actual strain tensor $\epsilon_{i j}(\mathbf{r})$ is linearly related to the local displacement vector $\mathbf{u}(\mathbf{r})$, which is obtained by solving the mechanical equilibrium condition.

The elastic strain energy density term $G_{v}^{e l}$ can be expressed as:

$$
G_{v}^{e l}=\int_{\epsilon_{i j}^{0}(\mathbf{r})}^{\epsilon_{i j}(\mathbf{r})} \sigma_{i j}(\mathbf{r}) d \epsilon_{i j}(\mathbf{r})
$$

where $\epsilon_{i j}^{0}(\mathbf{r})$ is the stress-free transformation strain, $\epsilon_{i j}(\mathbf{r})$ is the total strain and $\sigma_{i j}(\mathbf{r})$ is the elastic stress. We have considered isotropic elastic properties and hence $\sigma_{i j}(\mathbf{r})$ is given by:

$$
\sigma_{i j}(\mathbf{r})=\frac{E}{1+\nu}\left[\epsilon_{i j}^{e l}(\mathbf{r})+\frac{\nu}{1-2 \nu} \epsilon_{k k}^{e l}(\mathbf{r}) \delta_{i j}\right]
$$

where $E$ is Youngs modulus of elasticity and $\nu$ is Poisson ratio and $\epsilon_{k l}^{e l}(\mathbf{r})$ is the elastic strain, given by:

$$
\epsilon_{k l}^{e l}(\mathbf{r})=\epsilon_{k l}(\mathbf{r})-\epsilon_{k l}^{0}(\mathbf{r})-\epsilon_{k l}^{p l}(\mathbf{r})
$$

where $\epsilon_{k l}^{p l}(\mathbf{r})$ is the plastic strain.

The total strain $\epsilon_{i j}(\mathbf{r})$ is given by:

$$
\epsilon_{i j}(\mathbf{r})=\frac{1}{2}\left(\frac{\partial u_{i}(\mathbf{r})}{\partial r_{j}}+\frac{\partial u_{j}(\mathbf{r})}{\partial r_{i}}\right)
$$

where $\mathbf{u}(\mathbf{r})$ is the local displacement vector obtained by solving the mechanical equilibrium condition:

$$
\frac{\partial \sigma_{i j}(\mathbf{r})}{\partial r_{j}}=0
$$

The stress-free transformation strain is given by:

$$
\epsilon_{i j}^{0}(\mathbf{r})=\sum_{p=1}^{p=v} \eta_{p}(\mathbf{r}) \epsilon_{i j}^{0}(p)
$$

where $\epsilon_{i j}^{0}(p)$ is the stress-free transformation strain tensor corresponding to each variant [24] and is given by:

$$
\begin{aligned}
& \epsilon_{i j}^{0}(1)=\left[\begin{array}{lll}
\epsilon_{1} & \epsilon_{2} & \epsilon_{2} \\
\epsilon_{2} & \epsilon_{1} & \epsilon_{2} \\
\epsilon_{2} & \epsilon_{2} & \epsilon_{1}
\end{array}\right], \epsilon_{i j}^{0}(2)=\left[\begin{array}{ccc}
\epsilon_{1} & -\epsilon_{2} & -\epsilon_{2} \\
-\epsilon_{2} & \epsilon_{1} & \epsilon_{2} \\
-\epsilon_{2} & \epsilon_{2} & \epsilon_{1}
\end{array}\right], \\
& \epsilon_{i j}^{0}(3)=\left[\begin{array}{ccc}
\epsilon_{1} & -\epsilon_{2} & \epsilon_{2} \\
-\epsilon_{2} & \epsilon_{1} & -\epsilon_{2} \\
\epsilon_{2} & -\epsilon_{2} & \epsilon_{1}
\end{array}\right], \epsilon_{i j}^{0}(4)=\left[\begin{array}{ccc}
\epsilon_{1} & \epsilon_{2} & -\epsilon_{2} \\
\epsilon_{2} & \epsilon_{1} & -\epsilon_{2} \\
-\epsilon_{2} & -\epsilon_{2} & \epsilon_{1}
\end{array}\right]
\end{aligned}
$$

where $\epsilon_{1}=v_{s}+\frac{1}{3} v_{f}, \epsilon_{2}=\frac{1}{3} v_{f}, v_{s}=\frac{a_{\omega}}{\sqrt{2} \cdot a_{\beta}}-1$ and $v_{f}=\frac{2 c_{\omega}}{\sqrt{3} \cdot a_{\beta}}-\frac{a_{\omega}}{\sqrt{2} \cdot a_{\beta}}$. $a_{\beta}, a_{\omega}$ and $c_{\omega}$ are lattice constants of $\beta$ and $\omega$ phases, respectively. As stated 
earlier, the martensite variants-1, 2, 3 and 4 are governed by $\epsilon_{i j}^{0}(1), \epsilon_{i j}^{0}(2), \epsilon_{i j}^{0}(3)$ and $\epsilon_{i j}^{0}(4)$, respectively.

The plastic strain comes into existence only when the elastic stress exceeds the yield limit, thereby initiating plastic deformation that acts as a relaxation of the elastic stress. The von Mises criterion is employed to check whether the elastic stress has exceeded the yield stress $\left(\sigma_{y}\right)$. According to the von Mises criterion, plastic deformation occurs if:

$$
\begin{aligned}
\bar{\sigma}^{2}-\sigma_{y}^{2} & =\frac{1}{2}\left\{\left(\sigma_{x x}-\sigma_{y y}\right)^{2}+\left(\sigma_{y y}-\sigma_{z z}\right)^{2}\right. \\
& \left.+\left(\sigma_{z z}-\sigma_{x x}\right)^{2}\right\}+3\left(\sigma_{x y}^{2}+\sigma_{y z}^{2}+\sigma_{z x}^{2}\right)-\sigma_{y}^{2} \geq 0
\end{aligned}
$$

where $\bar{\sigma}$ is von Mises equivalent stress.

The evolution of the plastic strain field $[11,22,31]$ can be calculated by:

$$
\frac{\partial \epsilon_{i j}^{p l}(\mathbf{r})}{\partial t}=-k_{i j k l} \frac{\delta G_{v}^{\text {shear }}}{\delta \epsilon_{k l}^{p l}(\mathbf{r})}
$$

where $\epsilon_{i j}^{p l}(\mathbf{r})$ is the local plastic strain, $G_{v}^{\text {shear }}$ is shear energy density and $k_{i j k l}$ $\left(=k c_{i j k l}^{-1}\right)$ is plastic kinetic coefficient. $c_{i j k l}^{-1}$ denotes the compliance tensor and $k$ is a parameter, which controls the rate at which the elastic stresses are relaxed by means of plastic deformation and is called plastic relaxation rate.

The shear energy density $G_{v}^{\text {shear }}$ is given by:

$$
G_{v}^{\text {shear }}=\int_{e_{i j}^{0}(\mathbf{r})}^{e_{i j}(\mathbf{r})} c_{i j k l}\left(e_{k l}(\mathbf{r})-e_{k l}^{0}(\mathbf{r})\right) d e_{i j}(\mathbf{r})
$$

where $c_{i j k l}$ is the tensor of elastic constants, $e_{i j}(\mathbf{r})$ and $e_{i j}^{0}(\mathbf{r})$ are the deviatoric total strain tensor and deviatoric stress-free transformation strain tensors given by:

$$
\begin{gathered}
e_{i j}(\mathbf{r})=\epsilon_{i j}(\mathbf{r})-\frac{1}{3} \epsilon_{k k}(\mathbf{r}) \delta_{i j} \\
e_{i j}^{0}(\mathbf{r})=\epsilon_{i j}^{0 t o t}(\mathbf{r})-\frac{1}{3} \epsilon_{k k}^{0 t o t}(\mathbf{r}) \delta_{i j}
\end{gathered}
$$

where $\epsilon_{i j}^{0 t o t}(\mathbf{r})$ is given by:

$$
\epsilon_{i j}^{0 t o t}(\mathbf{r})=\epsilon_{i j}^{0}(\mathbf{r})+\epsilon_{i j}^{p l}(\mathbf{r})
$$

Linear isotropic strain hardening is considered by using the following expression [33]:

$$
\sigma_{y}=\sigma_{y}^{0}+H \epsilon^{p l}(\mathbf{r})
$$

where $\sigma_{y}$ is the yield stress of the material that depends on the plastic strain, $\sigma_{y}^{0}$ is the initial yield stress, $H$ is the hardening modulus and $\epsilon^{p l}(\mathbf{r})$ is the von Mises equivalent plastic strain. 


\subsection{Application of an external stress}

Under the application of an external stress, Eq. (2) has to be modified and the Gibbs energy of the system can be expressed as:

$$
G=\int_{V}\left(G_{v}^{\text {chem }}+G_{v}^{g r a d}+G_{v}^{e l}+G_{v}^{a p p l}\right) d V
$$

where $G_{v}^{a p p l}$ is the extra Gibbs energy density caused by the externally applied stress, expressed as:

$$
G_{v}^{a p p l}=-\sigma_{i j}^{a p p l} \epsilon_{i j}^{0}(\mathbf{r})
$$

where $\sigma_{i j}^{a p p l}$ is the externally applied Cauchy stress tensor, i.e.:

$$
\sigma_{i j}^{a p p l}=\left[\begin{array}{lll}
\sigma_{x x} & \sigma_{x y} & \sigma_{x z} \\
\sigma_{y x} & \sigma_{y y} & \sigma_{y z} \\
\sigma_{z x} & \sigma_{z y} & \sigma_{z z}
\end{array}\right]
$$

\section{Simulations}

Table 1 shows the input data for the simulations.

Table 1: Simulation data.

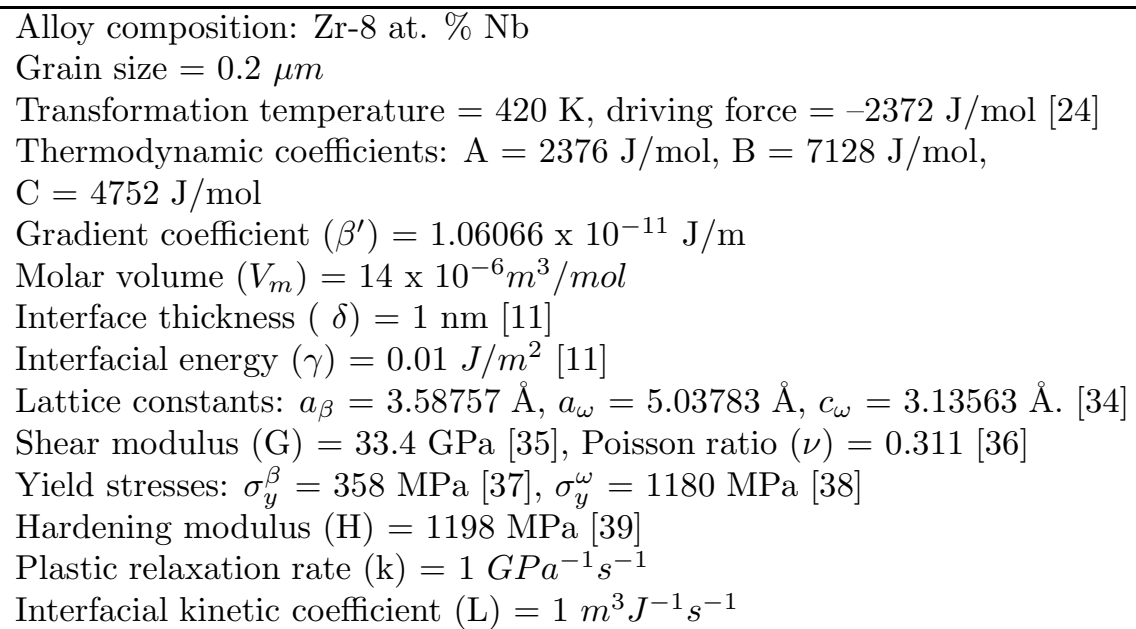

As the experimental data related to the mobility of the martensitic interface is ambiguous, the matrix of kinetic parameters $L_{p q}$ in Eq. (1) that governs the mobility of the martensitic interface is considered to be an identity matrix. The entire mathematical formulation is solved using tetrahedral finite elements and 
by using Dirichlet boundary conditions. Computations are performed on a mesh with 70x70x70 grid points.

We use random noise to initiate the nucleation process. In the first case, a simulation is performed to study the omega phase formation under athermal quench conditions. In order to study the effects of plasticity, another simulation is performed under athermal quench conditions but without plasticity, i.e. by enormously increasing the yield limit of the two phases such that the von Mises equivalent stresses can never exceed the yield limit. In order to study the effects of stress-states on the omega phase formation, six different simulations are performed under different stress states (Eq. (21)), such as uni-axial tensile $\left(\sigma_{x x}=1 \mathrm{GPa}\right)$, uni-axial compressive $\left(\sigma_{x x}=-1 \mathrm{GPa}\right)$, bi-axial tensile $\left(\sigma_{x x}=\right.$ $\left.\sigma_{y y}=1 \mathrm{GPa}\right)$, bi-axial compressive $\left(\sigma_{x x}=\sigma_{y y}=-1 \mathrm{GPa}\right)$, shear $\left(\sigma_{x y}=1\right.$ $\mathrm{GPa})$ and tri-axial $\left(\sigma_{x x}=1 \mathrm{GPa}, \sigma_{y y}=750 \mathrm{MPa}, \sigma_{z z}=-500 \mathrm{MPa}\right)$. In order to study the effect of stress loading direction on the omega phase formation, three different simulations are performed by changing the direction of shear loading $\left(\sigma_{x y}\right.$ or $\sigma_{y z}$ or $\left.\sigma_{z x}=1 \mathrm{GPa}\right)$.

\section{Results and discussion}

\subsection{Microstructure evolution during athermal transformation}

The microstructure evolution during athermal transformation is shown in Fig. 1. The iso-surfaces of the four phase-field variables $\left(\eta_{p}=0.5\right)$, i.e. the omega variants-1, 2, 3, 4, are shown in red, blue, green and yellow, respectively. The random noise generates some randomly shaped omega particles (Fig. 1a). As the transformation progresses, the randomly shaped nuclei transform into ellipsoidal shaped particles (Figs. 1b-d), mainly due to the elastic energy. The number of omega particles increases due to the chemical driving force as well as due to the autocatalytic effect, i.e. self nucleation of new omega particles. Autocatalysis occurs in order to minimize the elastic stresses created by the growing omega particles. Some omega particles merge together and form into a bigger omega particle (marked by circles in Fig. 1d). These merged omega particles could potentially lead to embrittlement of the material, as they become barriers for dislocation motion [5]. The simulated microstructure shows that all the omega variants are aligned along the $\langle 111\rangle_{\beta}$ direction. The morphology and alignment of the omega particles are in good agreement with experimental results $[3,4]$.

All four omega variants are formed, although variants- 2 and 4 seem to be slightly more favored over variants- 1 and 3 (Fig. 1e). Although the initial growth conditions are identical for all the variants, the internal stresses can either increase or decrease the net available driving force for formation of each variant, depending on the respective orientation and the transformation strain tensor.

The 2D section plots of microstructure, von Mises equivalent stress, strain and plastic strain plotted on $(111)^{\gamma}$ are shown in Fig. 2. Fig. 2a shows the four crystallographic variants of the omega phase in orange, yellow, green and 


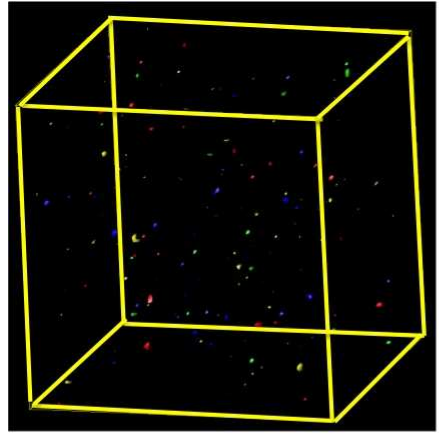

(a)

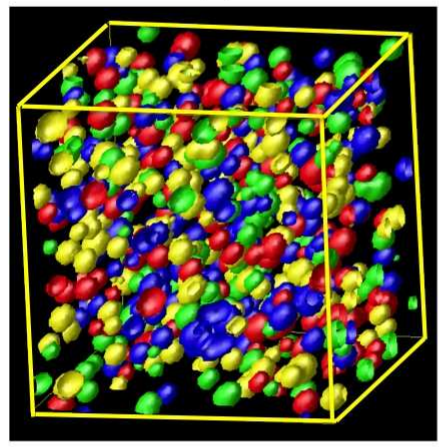

(c)

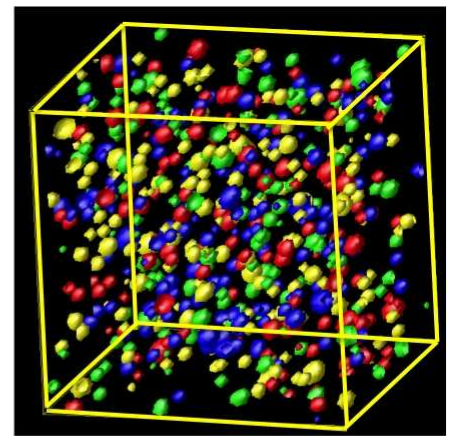

(b)

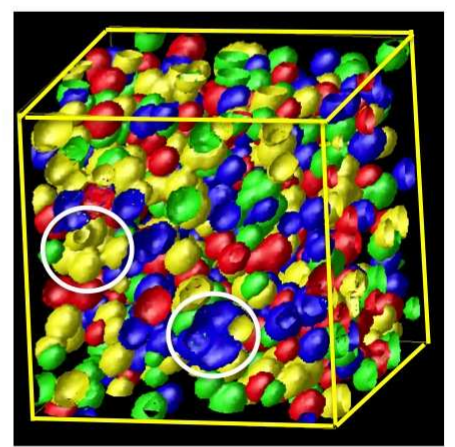

(d)

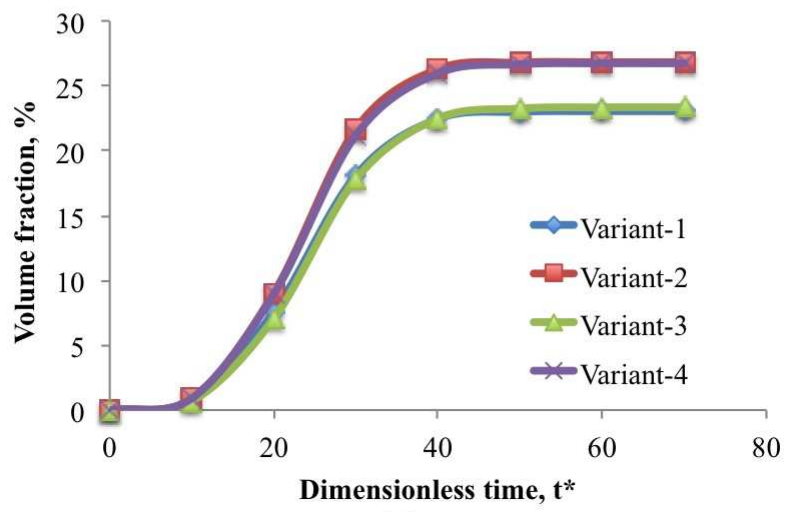

(e)

Figure 1: Athermal microstructure evolution at (a) $t^{*}=0(b) t^{*}=10(c) t^{*}=15$ (d) $t^{*}=20$ and (e) variation in volume fraction of different variants with dimensionless time $t^{*}$. Iso-surfaces of the phase-field variables (four omega variants-1, 2, 3, 4) are shown in red, blue, green and yellow, respectively. Fine ellipsoidal omega particles form and are aligned along the $\langle 111\rangle_{\beta}$ direction. Volume fractions of variants-2, 4 are slightly more than those of variants- 1,3 .

blue colors. As the omega phase is relatively much harder than the beta phase, the formation of omega particles increases the stresses in those regions and 
hence the equivalent stress in the omega particles is higher compared to the surrounding matrix (Fig. 2b). Due to these high stresses, the strains (Fig. 2c) and plastic strains (Fig. 2d) in the omega particles are also higher compared to the parent phase. Although most of the omega particles give rise to high stresses and strains (Figs. 2b,c), only some of the omega particles give rise to plastic deformation (Figs. 2a,d). As the yield stress of the omega phase is very high $(1180 \mathrm{MPa})$, plastic deformation inside the omega particles occurs only in areas where the internal stresses exceed this yield limit and hence only some omega particles contribute to the plastic deformation of the material.

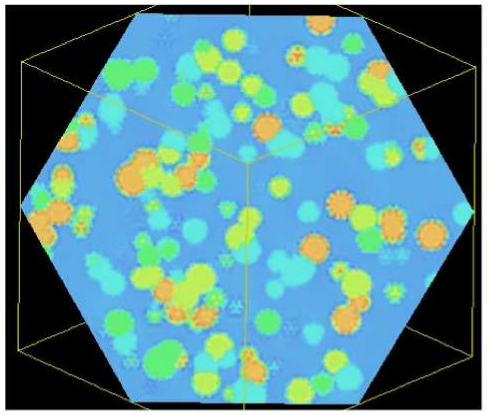

(a)

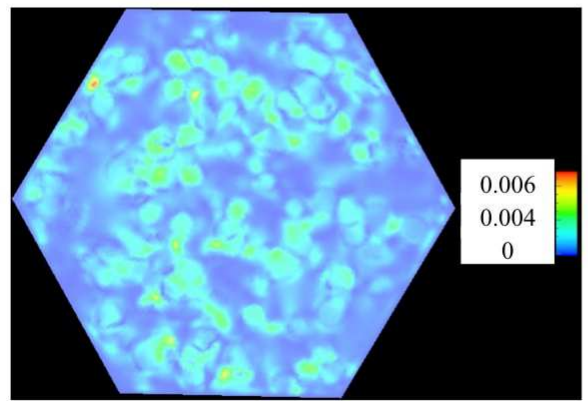

(c)

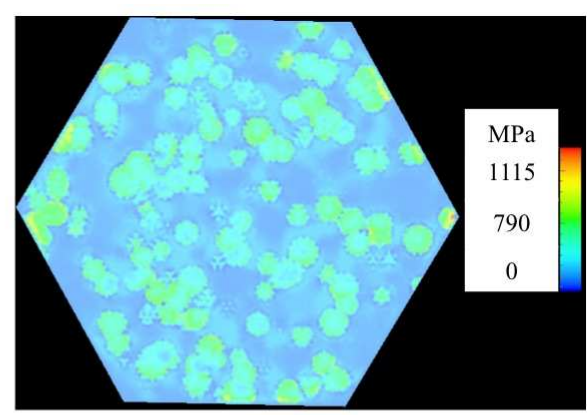

(b)

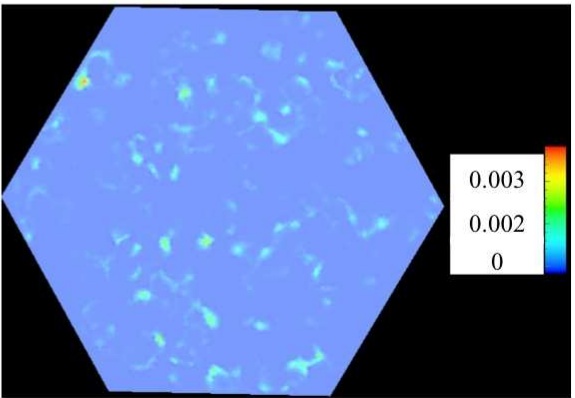

(d)

Figure 2: 2D section plots of (a) microstructure (b) von Mises equivalent stress (c) von Mises equivalent strain (d) von Mises equivalent plastic strain, plotted on (111) $\gamma$. Formation of omega particles increases the stresses and strains in those regions, although only some omega particles give rise to plastic deformation.

\subsection{Effect of plasticity on omega phase formation}

The microstructure evolution in the absence of plasticity is shown in Fig. 3. The evolution of the omega phase does not show a large difference in the two cases, i.e. with and without plasticity (Figs. 1, 3 and 4a). However, omega phase forms slightly more readily in the presence of plasticity compared to that in its absence (Fig. 4a). The stress-strain curves (Fig. 4b) show that the phase transformation in the absence of plasticity leads to a large stress. Due 
to the lack of relaxation by means of plastic deformation, the internal stresses are large in this case. Such high internal stresses act as a barrier and inhibit the transformation. Hence the phase transformation is slightly less favorable in the absence of plasticity compared to that in its presence, where the stresses are relaxed by plastic deformation and the growth of omega phase is facilitated. The omega phase formation in the presence of plasticity leads to slightly larger elongation (equivalent strain) compared to that in its absence (Fig. 4b). As the plastic strains (dislocations) increase the ductility of a material, up to certain extent, the presence of plasticity leads to slightly larger elongation.

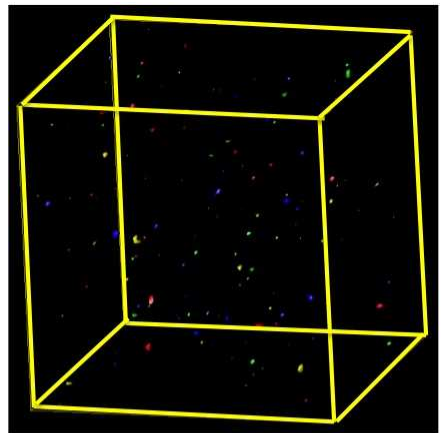

(a)

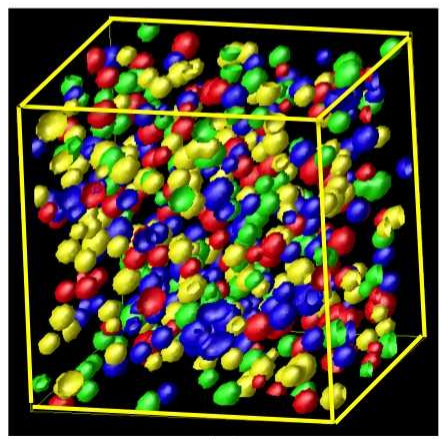

(c)

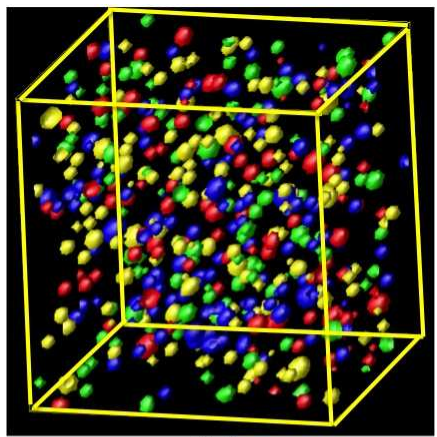

(b)

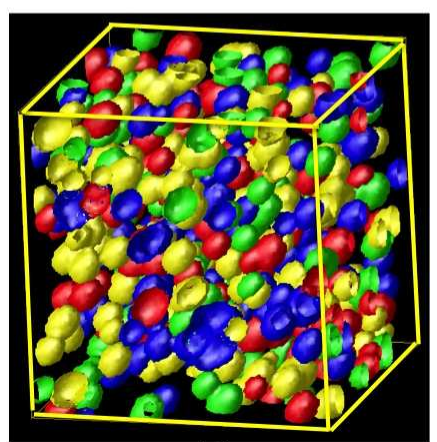

(d)

Figure 3: Athermal microstructure evolution in the absence of plasticity, at (a) $t^{*}=0$ (b) $t^{*}=$ 10 (c) $t^{*}=15(d) t^{*}=20$. Omega phase formation is slightly less favorable in the absence of plasticity compared to that in its presence (Fig. 1).

\subsection{Effect of external stresses on omega phase formation and on the mechanical properties}

The microstructures obtained at $t^{*}=15$ under different stress states are shown in Fig. 5. The differences in the volume fraction of omega phase (Figs. $5 \mathrm{a}-\mathrm{f}$ ) as well as the variant selection (Figs. $5 \mathrm{~d}$, e) can be seen. The evolution of omega phase is different under different stress states (Fig. 6a). The omega phase forms relatively more readily under bi-axial compressive loading compared to 


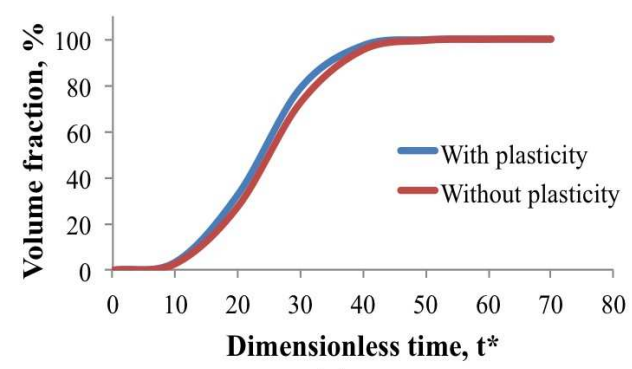

(a)

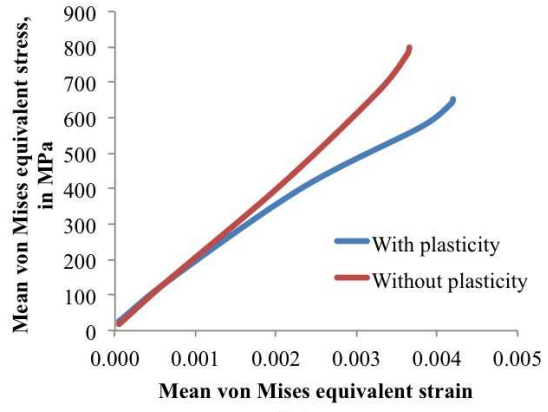

(b)

Figure 4: Comparison of the (a) evolution of omega phase (b) von Mises equivalent stressstrain curves, in the presence and in the absence of plasticity. In the absence of plasticity, evolution of omega phase is slightly less favorable due to lack of stress relaxation and hence leads to larger stress compared to that in the presence of plasticity.

that under other stress states, especially under bi-axial tensile loading. As the additional mechanical energy due to the applied stress is an algebraic addition (Eq. (19)) to the total Gibbs energy the applied stress, depending on whether it is tensile or compressive, will either increase or decrease the net available driving force. In the case of uni-axial tensile loading, the additional mechanical energy is a product of the applied tensile stress $\sigma_{x x}=1 \mathrm{GPa}$ and the transformation strain $\epsilon_{x x}^{0}(p)=-0.0016$, which retracts from the net driving force and hence does not favor the formation of omega phase. However, in the case of uni-axial compressive loading, the additional mechanical energy (Eq. (20)) is a product of the applied compressive stress $\sigma_{x x}=-1 \mathrm{GPa}$ and the transformation strain $\epsilon_{x x}^{0}(p)=-0.0016$, which adds up to the net driving force and favors the formation of omega phase. Hence the omega phase forms relatively more readily under compressive loading compared to that under tensile loading. This trend is observed under both uni-axial and bi-axial stress states.

Fig. 6b shows the stress-strain plots obtained under different stress states. The maximum stress is obtained under shear loading, whereas the maximum elongation (equivalent strain) is obtained under tri-axial loading. Figs. 6c, d show that as the volume fraction of omega increases, the internal stresses and the extent of plastic deformation increase. Due to the increasing volume fraction of omega phase, the internal stresses increase and exceed the yield limit, thereby increase the extent of plastic deformation. The maximum plastic deformation is obtained under tri-axial loading (Fig. 6d). These results show that the differences in the microstructure evolution under different stress states lead to the variations in the final mechanical properties of $\mathrm{Zr}-\mathrm{Nb}$ alloys.

\subsection{Effect of stress loading direction on omega phase formation}

Under some loading conditions, the loading direction plays an important role in variant selection. The difference in variant selection under shear loading along xy, yz and zx directions is shown in Fig. 7. The extra Gibbs energy term 


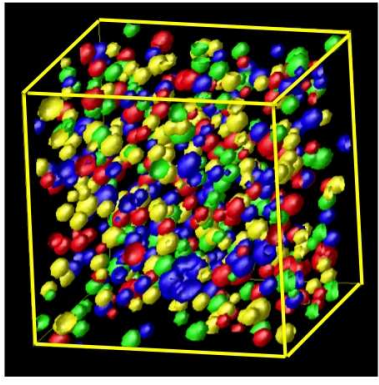

(a)

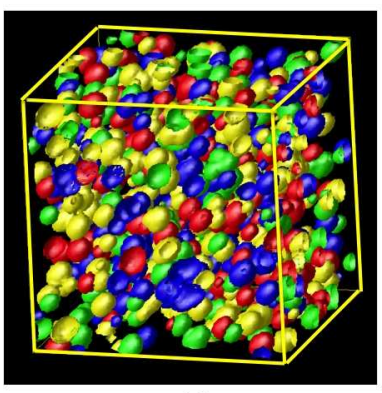

(d)

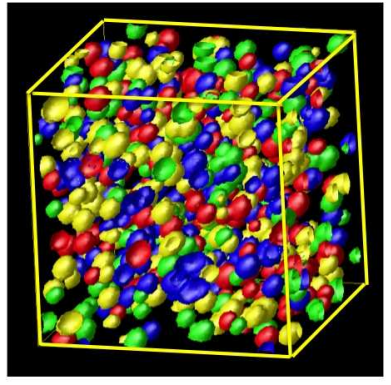

(b)

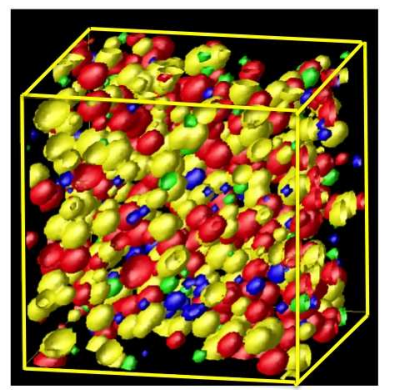

(e)

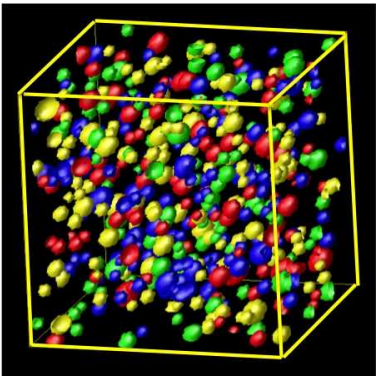

(c)

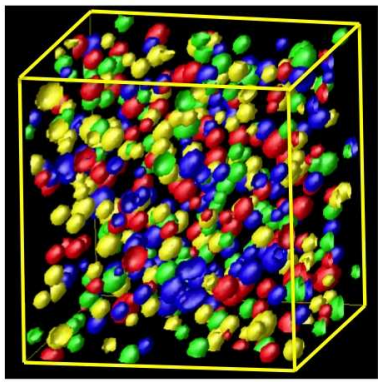

(f)

Figure 5: Microstructures obtained at $t^{*}=15$ under (a) uni-axial tensile (b) uni-axial compressive (c) bi-axial tensile (d) bi-axial compressive (e) shear (f) tri-axial stress states. Microstructure evolution as well as variant selection is different under different stress states.

due to the applied stress is a product of applied stress and the transformation strain in that direction (Eq. (20)), which either adds up to or retracts from the net Gibbs energy.

In the case of $\tau_{x y}$ loading, the additional mechanical energy is a product of applied shear stress $\tau_{x y}=1 \mathrm{GPa}$ and transformation strain $\epsilon_{x y}^{0}(p)$ of the corresponding variant. Variants - 1, 4 have a transformation strain of 0.00543 , whereas variants $-2,3$ have a transformation strain of -0.00543 along xy direction. Thus the mechanical energy contribution due to variants - 1, 4 adds up to, whereas the contribution due to variants - 2,3 retracts from, the net driving force. Hence variants - 1, 4 are favored over variants - 2, 3 under $\tau_{x y}$ loading. Thus depending on the loading direction and the sign of the transformation strain, formation of some variants will be favored over that of others. Hence variants-1, 4 are favored under $\tau_{x y}$, variants-1, 2 are favored under $\tau_{y z}$ and variants-1, 3 are favored under $\tau_{z x}$ (Fig. 7). Moreover, variant-1 is favored under all three shear loadings. In the case of tensile and compressive loadings (both uni-axial and bi-axial), the transformation strains are equal and of same nature (tensile) in all the three directions. Hence, we have not observed any directional dependence of variant selection under tensile and compressive loadings. 


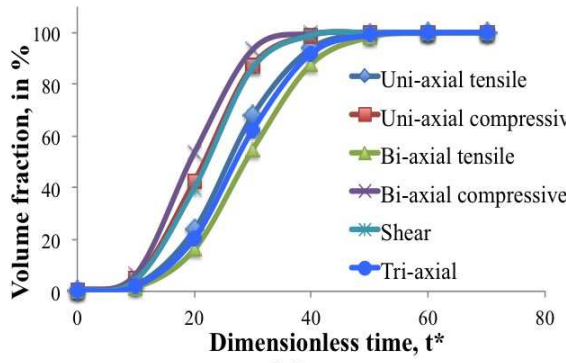

(a)

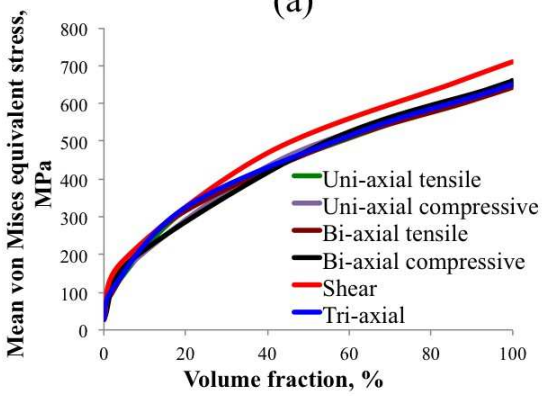

(c)

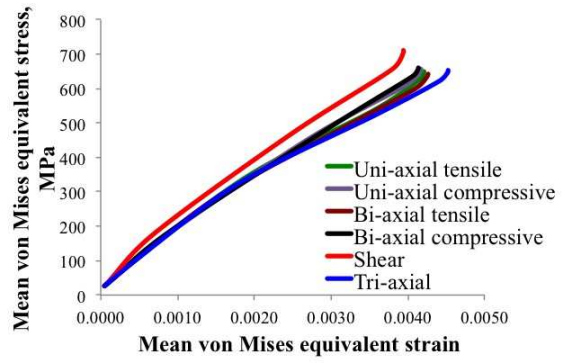

(b)

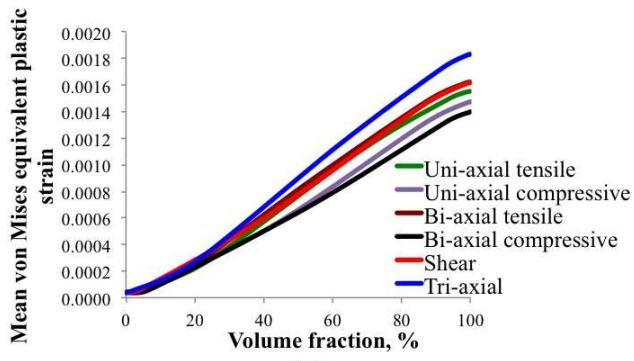

(d)

Figure 6: Variations in (a) evolution of volume fraction with t* (b) von Mises equivalent stress-strain curves (c) volume fraction with von Mises equivalent stress (d) volume fraction with von Mises equivalent plastic strain, under different stress states. Omega phase forms more readily under bi-axial compressive loading compared to that under other stress states. The variations in the microstructure evolution and the variant selection has given rise to the variations in the mechanical properties of the material.

\section{Conclusions}

Our results indicate that during the athermal $\beta \rightarrow \omega$ phase transformation, omega particles grow into ellipsoidal shaped units, mainly due to the elastic energy. As the transformation progresses, the number of omega particles increases, due to the chemical driving force and the autocatalytic effect. We observe that all the omega variants are aligned along the $\langle 111\rangle_{\beta}$ direction, which is in good agreement with experimental results $[3,4]$. Although the initial growth conditions are identical for all the variants, the internal stresses can either increase or decrease the net available driving force and can favor the formation of some variants over that of others.

Due to stress relaxation by means of plastic deformation, the omega phase forms slightly more readily in the presence of plasticity compared to that in its absence. In the absence of plasticity, the large internal stresses developed inside the material inhibit the transformation. The presence of plasticity leads to slightly larger elongation of the material, compared to that in its absence, indicating increased ductility.

The microstructure evolution is different under different stress states, such as uni-axial tensile and compressive, bi-axial tensile and compressive, shear and 


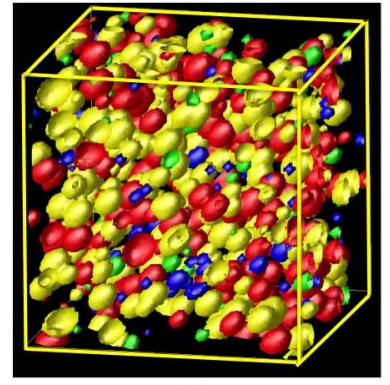

(a)

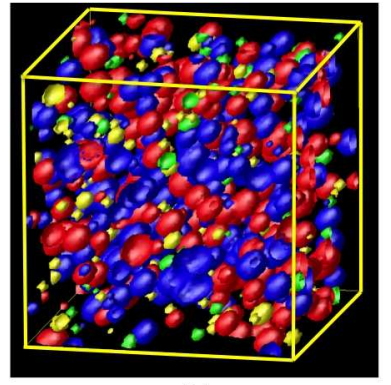

(b)

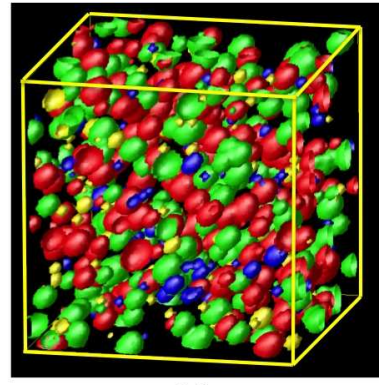

(c)

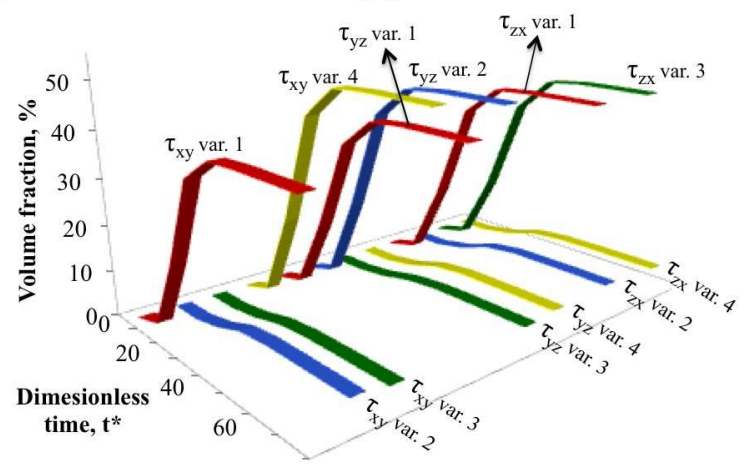

(d)

Figure 7: Microstructures formed at $t^{*}=15$ under shear loading along (a) xy (b) yz (c) zx directions, (d) variation in volume fractions of different variants, with $\mathrm{t}^{*}$, under different shear loading directions. Variants-1, 4 under $\tau_{x y}$, variants-1, 2 under $\tau_{y z}$ and variants-1, 3 under $\tau_{z x}$ are favored.

tri-axial loadings. As the additional mechanical energy due to the applied stress is an algebraic addition to the total Gibbs energy, the nature of the applied stress will either increase or decrease the net available driving force. We observe that the omega phase forms more readily under compressive loading compared to that under tensile loading.

The co-evolution of microstructure and mechanical properties is also studied. The maximum stress is obtained under shear loading, whereas the maximum elongation (equivalent strain) and plastic deformation are obtained under triaxial loading. The differences in the microstructure evolution under different stress states lead to the variations in the mechanical properties of $\mathrm{Zr}-\mathrm{Nb}$ alloys.

Our model predicts that the stress loading direction plays an important role in variant selection. Three different shear loading directions are studied and the results indicate that variants- 1,4 are favored under $\tau_{x y}$, variants- 1,2 are favored under $\tau_{y z}$ and variants-1,3 are favored under $\tau_{z x}$. These differences are attributed to the additional mechanical energy due to the applied stress, which either adds up to or retracts from the net Gibbs energy depending on the nature of the transformation strain of the corresponding variant and thereby 
favors some variants over the others.

As Zr-Nb alloys play an important role in the chemical and nuclear industries as well as due to their usage in medical implants, it is essential to predict the microstructure evolution and mechanical properties during phase transformations in order to predict the product behavior under different thermo-mechanical conditions. Our results show that the omega phase formation is affected by varying the processing conditions (stress states and loading directions) and hence high-

light the need for thorough in-situ microstructural characterization works on Zr-Nb alloys.

\section{Acknowledgements}

This work was supported by the U.S. Department of Energy. This research used resources provided by the Los Alamos National Laboratory Institutional Computing Program, which is supported by the U.S. Department of Energy.

\section{References}

[1] R. Kondo, N. Nomura, Suyalatu, Y. Tsutsumi, H. Doi, T. Hanawa, Acta Biomater. 7 (2011) 4278-4284.

[2] S. Zhang, X. Zhang, Y. Zhu, S. Zhang, L. Qi, R. Liu, Comp. Mat. Sci. 61 (2012) 42-49.

[3] R. Tewari, D. Srivastava, G.K. Dey, J.K. Chakravarty, S. Banerjee, J. Nucl. Mat. 383 (2008) 153-171.

[4] S. Banerjee, R. Tewari, G.K. Dey, Int. J. Mat. Res. 97 (2006) 963-977.

[5] D. Ping, Acta Metall. Sin. 27 (2014) 1-11.

[6] M.X. Huang, O. Bouaziz, D. Barbier, S. Allain, J. Mater. Sci. 46 (2011) 7410-7414.

[7] H. Hallberg, P. Håkansson, M. Ristinmaa, Int. J. Plast. 23 (2007) 12131239.

[8] L.Q. Chen, Annu. Rev. Mater. Res. 32 (2002) 113-140.

[9] S. Minamoto, S. Nomoto, A. Hamaya, T. Horiuchi, S. Miura, ISIJ Int. 50 (2010) 1914-1919.

[10] A.G. Khachaturyan, Theory of Structural Transformations in Solids, John Wiley and Sons, New York, 1983.

[11] H.K. Yeddu, A. Malik, J. Ågren, G. Amberg, A. Borgenstam, Acta Mater. 60 (2012) 1538-1547.

[12] S.R. Shenoy, T. Lookman, A. Saxena, A.R. Bishop, Phys. Rev. B 60 (1999) R12537-12541. 
[13] Y. Wang, A.G. Khachaturyan, Acta Mater. 45 (1997) 759-773.

[14] H.K. Yeddu, A. Borgenstam, P. Hedström, J. Ågren, Mater. Sci. Eng. A 538 (2012) 173-181.

[15] A. Artemev, Y. Jin, A.G. Khachaturyan, Acta Mater. 49 (2001) 1165-1177.

[16] H.K. Yeddu, A. Borgenstam, J. Ågren, J. Alloys Compd. 577S (2013) S141S146.

[17] A. Malik, H.K. Yeddu, G. Amberg, A. Borgenstam, J. Ågren, Mater. Sci. Eng. A 556 (2012) 221-232.

[18] R. Ahluwalia, T. Lookman, A. Saxena, R.C. Albers, Acta Mater. 52 (2004) 209-218.

[19] H.K. Yeddu, A. Borgenstam, J. Ågren, Acta Mater. 61 (2013) 2595-2606.

[20] R. Ahluwalia, T. Lookman, A. Saxena, Acta Mater. 54 (2006) 2109-2120.

[21] H.K. Yeddu, V.I. Razumovskiy, A. Borgenstam, P.A. Korzhavyi, A.V. Ruban, J. Ågren, Acta Mater. 60 (2012) 6508-6517.

[22] A. Yamanaka, T. Takaki, Y. Tomita, Mater. Sci. Eng. A 491 (2008) 378-384.

[23] H.K. Yeddu, T. Lookman, A. Saxena, Acta Mater. 61 (2013) 6972-6982.

[24] B. Tang, Y.W. Cui, H. Chang, H. Kou, J. Li, L. Zhou, Comp. Mat. Sci. 53 (2012) 187-193.

[25] H.K. Yeddu, T. Lookman, A. Saxena, J. Mater. Sci. 49 (2014) 3642-3651.

[26] T.W. Heo, L.Q. Chen, Acta Mater. 76 (2014) 68-81.

[27] H.K. Yeddu, T. Lookman, A. Saxena, Mater. Sci. Eng. A 594 (2014) 48-51.

[28] M. Mamivand, M.A. Zaeem, H.E. Kadiri, Int. J. Plast. 60 (2014) 71-86.

[29] H.K. Yeddu, T. Lookman, A. Borgenstam, J. Ågren, A. Saxena, Mater. Sci. Eng. A 608 (2014) 101-105.

[30] J. Kundin, D. Raabe, H. Emmerich, J. Mech. Phys. Solids 59 (2011) 20822102.

[31] X.H. Guo, S.Q. Shi, X.Q. Ma, Appl. Phys. Lett. 87 (2005) 221910.

[32] S.M. Allen, J.W. Cahn, Acta Metall. 27 (1979) 1085-1095.

[33] E.A. De souza Neto, D. Peric, D.R.J. Owen, Computational methods for plasticity theory and applications, John Wiley, Chichester, 2008.

[34] G. Aurelio, A.F. Guillermet, G.J. Cuello, J, J. Nucl. Mat. 341 (2005) 1-11. 
[35] G. Grimvall, Thermophysical Properties of Materials, first ed., Elsevier, Amsterdam, 1999.

[36] W. Liu, B. Li, L. Wang, J. Zhang, Y. Zhao, J. Appl. Phys. 104 (2008) 076102 .

[37] S. Cai, M.R. Daymond, R.A. Holt, Acta Mater. 60 (2012) 3355-3369.

[38] Y. Zhao, J. Zhang, Appl. Phys. Lett. 91 (2007) 201907.

[39] S.R. Chen, G.T. Gray III, J. Phys. IV France. 7 (1997) C3-741-746. 